The Use of a New Potentiostat/Coulometer in the Controlled-Potential Coulometric Determination of Plutonium

Paul G. Mendoza, Jr.

Donald J. Temer

Darryl D. Jackson 


\title{
The Use of a New Potentiostat/Coulometer in the Controlled-Potential Coulometric Determination of Plutonium
}

\author{
by \\ Paul G. Mendoza, Jr., Donald J. Temer, \\ and Darryl D. Jackson
}

\begin{abstract}
We evaluated a PAR 273 potentiostat and PAR 279 coulometer using iron as a stand-in for plutonium. Over $\mathbf{4 0 0}$ runs consisting of electrical checks, blanks, and iron solutions were made. Manual operation of the instrumentation produced precisions better than $0.1 \%$. Computer control of the instrumentation using a PC and a program written in QuickBASIC produced precisions of about $0.12 \%$. The computer program automatically set up and controlled the instrumentation and recorded the data. The potentiostat's current autoranging function caused erratic coulometer results. Use of the PAR 273's low-pass filter (LPF) removed the amperometric endpoint current noise, but it also biased the current. The PAR 273A's LPF filtered the noise without introducing a bias. The coulometer functions, such as current integration and communication interface, occasionally locked up after a current overrange condition. The occasional procedural error revealed that the potentiostat's ability to produce $>1 \mathrm{~A}$ current can ruin the coulometer's input circuitry, which is protected up to $0.2 \mathrm{~A}$ for current ranges $<1 \mathrm{~A}$.
\end{abstract}

\section{Introduction}

\section{A. Significance of Coulometry}

Controlled potential coulometry is one of the techniques of choice for the precise assay of plutonium ${ }^{1-3}$ because of the high precision, accuracy, and robustness attainable. Precisions of $0.1 \%$ relative standard deviation (RSD) or better are common. In $0.5 \mathrm{M}$ sulfuric acid, the plutonium redox couple used, $\mathrm{Pu}(\mathrm{III}) / \mathrm{Pu}(\mathrm{IV})$, makes for a selective analysis. Indeed, of the common contaminants found in plutonium, only iron is found to 
interfere. In addition, the technique is reliable and the laboratory manipulations are not overly complex.

This report describes our work in the evaluation of a new controlled-potential coulometry instrument that can be used in the analysis of plutonium.

\section{B. Description of the Method}

At Los Alamos ${ }^{4}$ the electrolysis is done in a three-electrode cell in $0.5 \mathrm{M}$ sulfuric acid electrolyte. The cylindrical working electrode is made of platinum mesh, and the counter, or auxiliary, electrode is a helical platinum wire. The reference electrode is a saturated calomel electrode. Each electrode half-cell is separated from the other two by bridge tubes with porous glass tips.

Under inert gas, with efficient stirring, the samples are first reduced to $\mathrm{Pu}(\mathrm{III})$ at $0.310 \mathrm{~V}$ to a $30-\mu \mathrm{A}$ amperometric end-point and then oxidized to $\mathrm{Pu}(\mathrm{IV})$ at $0.67 \mathrm{~V}$, also to a $30-\mu \mathrm{A}$ end-point. It is this second, anodic current that is measured with a coulometer and used as a measure of the plutonium. New Brunswick Laboratory certified reference material plutonium standards and $0.5 \mathrm{M}$ sulfuric acid blanks are electrolyzed in a similar fashion during an analysis. The iron determined by a spectrophotometric method is used to correct the plutonium result.

The potentiostat and coulometer we have traditionally used at Los Alamos are the PAR 173 and PAR 179." The current is monitored from the $I / E$ converter of the 173 with a digital voltmeter. The method routinely produces precisions better than $0.1 \%$ RSD.

Recently the PAR electrochemical instrumentation has been updated from the 100 to the 200 series. The new PAR 273 potentiostat has its own microprocessor for control. Alternatively it can be connected to an external controlling computer by a GPIB port. The design incorporates extensive computer control with over 100 commands available to perform a variety of electrochemical techniques, such as coulometry (using the PAR 273's internal coulometer) or cyclic voltammetry. The PAR 279 coulometer can be computer controlled through an RS232 port. It also has available a number of commands to control the instrument and to obtain data, although the current range still has to be set manually.

\section{Rationale}

Our preliminary evaluation of the 200 series suggested that using the newer instrumentation may be beneficial to our analytical work. Our interest was driven by the need to find a replacement for the discontinued PAR 179 coulometer.

We found the computer-control ability of the 200 series useful in that it could impart a degree of automation to the analysis by removing the analyst from the instrument setup, control, and data recording. The additional electrochemical techniques that the newer instrumentation offered fit in with future projects we are contemplating.

\section{Methodology}

Our study was divided into two parts. For Part 1, we evaluated the PAR 273/279 with manual control of the instrument. Part 2 was to evaluate the instrument under computer control. We used the performance of the 100 series instrumentation, which we have used extensively for years, as the bench mark for comparison with the newer

\footnotetext{
${ }^{*}$ EG\&G PARC, P.O. Box 2565, Princeton, NJ 08618.
} 
instrument, that is, how does the $273 / 279$ compare with the $173 / 179$. The criteria we used were

- Precision

- Accuracy

- Robustness.

\section{A. Reagents}

The $0.5 \mathrm{M} \mathrm{H}_{2} \mathrm{SO}_{4}$ supporting electrolyte was made up in an 8 - $\mathrm{L}$ batch with reagent-grade sulfuric acid and deionized water. After the first batch was exhausted, a second 8-L batch was made with the same acid but with water from a Corning glass still. A small batch of electrolyte was prepared with Seastar ultrapure sulfuric acid and deionized water.

Iron solution standard was prepared from reagent-grade $\mathrm{FeSO}_{4} \cdot 7 \mathrm{H}_{2} \mathrm{O}$ in $0.5 \mathrm{M}$ sulfuric acid.

Saturated $\mathrm{KCl}$, argon, and deionized or glass-distilled water were also used.

\section{B. Equipment and Procedures}

Teflon squeeze bottles were used to contain water and electrolyte. The electrolyte bottle was used to triple-rinse the electrodes between runs and to triple-rinse the cell bridge tubes at the start of a series of runs. Between rinses the extra electrolyte was aspirated from the electrodes. We occasionally cleaned the working and counter electrodes by leaving them immersed in hot nitric acid overnight.

A Teflon dropping bottle was used for weight-aliquoting the iron solution into the electrolysis cells. A 5-place electronic balance was used for weighing the dropping bottle. Static was removed with a 210 Po source.

A 9-L Pyrex bottle contained the electrolyte. Electrolyte from the first batch was dispensed by a stopcock-controlled siphon to a $25-\mathrm{mL}$ mark on the cell. Dispensing from the second batch of electrolyte, prepared for Part 2, was done with an electronic burette.

The electrolysis cell was a slightly modified PAR model 9600 coulometry cell system with argon purge. The modification for Part 1 was the use of the helical platinum wire counter electrode. For Part 2, we also used this kind of electrode along with cells that had the central contact removed and the resulting hole sealed. For electrical checks we used a $99.27-\Omega, 10-\mathrm{W}$ resistor placed in the electrometer reference plug with the counter and working electrodes clips across the resistor.

The PAR 273 potentiostat settings were as follows:

$$
\begin{aligned}
E / I 1: & 0.310 \mathrm{~V} \\
\text { Delay 1 and 2: } & 999 \mathrm{~s} \\
\text { Scan 1 and 2: } & \text { Step } \\
\text { E/I 2: } & 0.670 \mathrm{~V} \\
\text { Current range: } & 100 \mu \mathrm{A}
\end{aligned}
$$

For electrical checks, E/I 1 and Delay 1 were set to (), and Delay 2 was set to $600 \mathrm{~s}$. The PAR 279 settings were as follows: 
Functions: anodic, net

Current range: $100 \mathrm{~mA}$ for samples, $10 \mathrm{~mA}$

for blanks and electrical checks

For this study iron was used as a stand-in for plutonium. Blanks and samples were reduced until the 273 current display showed $30 \mu \mathrm{A}$, then the Advance was pushed to start the oxidation. For samples the oxidation was continued to $30 \mu \mathrm{A}$, whereas for blanks it was continued for $8 \mathrm{~min}$ while the coulombs from the 279 read-out were recorded every minute. Data recorded were the actual reduction and oxidation potentials and times as well as the amount of anodic current. A day's run proceeded with a starting electrical check, a minimum of 3 blanks, the iron solutions, a final blank, and a final electrical check.

For Part 2, the instrumentation and procedure were similar to that described above, the main difference being that a computer program (PARPU) performed the setup, control, and data recording. Two different potentiostats, a PAR 273 and a $273 \mathrm{~A}$, were used. Setup and control differences were related to the potentiostat current range. During electrolysis PARPU lowered the starting current range $(100 \mathrm{~mA}$ for samples and $10 \mathrm{~mA}$ for blanks) by a factor of 100 when the current reached $0.5 \%$ of the setup current range. The current monitored for the end-point determination was low-pass filtered by the potentiostat. Data recording differed in several respects: the 279 cathodic function was also set to measure the amount of reduction current, the actual reduction and oxidation potentials were not recorded, and in addition the coulombs from the internal coulometer included in the 273 were recorded.

We obtained coulogram curve data by reducing the same iron solution to $0.150 \mathrm{~V}$ and then measuring the coulombs from the anodic currents produced by $50-\mathrm{mV}$ increases of the potential. The currents were integrated to the $30-\mu \mathrm{A}$ end point. Cathodic currents were measured in a similar fashion with the iron oxidized to $0.700 \mathrm{~V}$ before each $50-\mathrm{mV}$ decrease.

An IBM-XT PC with an EGA card and National Instruments" GPIB PCIIA card was used for Part 2. The PC was connected to the potentiostat via the GPIB port and to the 279 via a serial port. To aid in debugging, the potentiostat was also connected to a "dumb" terminal via the serial port to monitor the GPIB bus activity. Switch and software settings appear in Appendix A.

\section{Results and Discussion}

For this study we ran 419 runs, including electrical checks, blanks, and iron solution electrolysis. We think this number of runs is sufficient to give us an understanding of the capability and performance of the newer instrumentation.

\section{A. Precision and Accuracy}

Figures 1 and 2 show the coulogram curve 5 for our electrochemical system. The $0.413-\mathrm{V}$ formal potential (VS SCE) was calculated from the intersection of the straight lines fit to linear points of Fig. 2. Using the Nernst equation, we estimate that at $0.310 \mathrm{~V}$ about $98 \%$ of the iron was in the ferrous state. By not doing an exhaustive reduction, we are able to test the potentiostat's ability to hold a selected potential.

\footnotetext{
* National Instruments, 654 Bridge Point Parkway, Austin, TX 787.30-5039.
} 


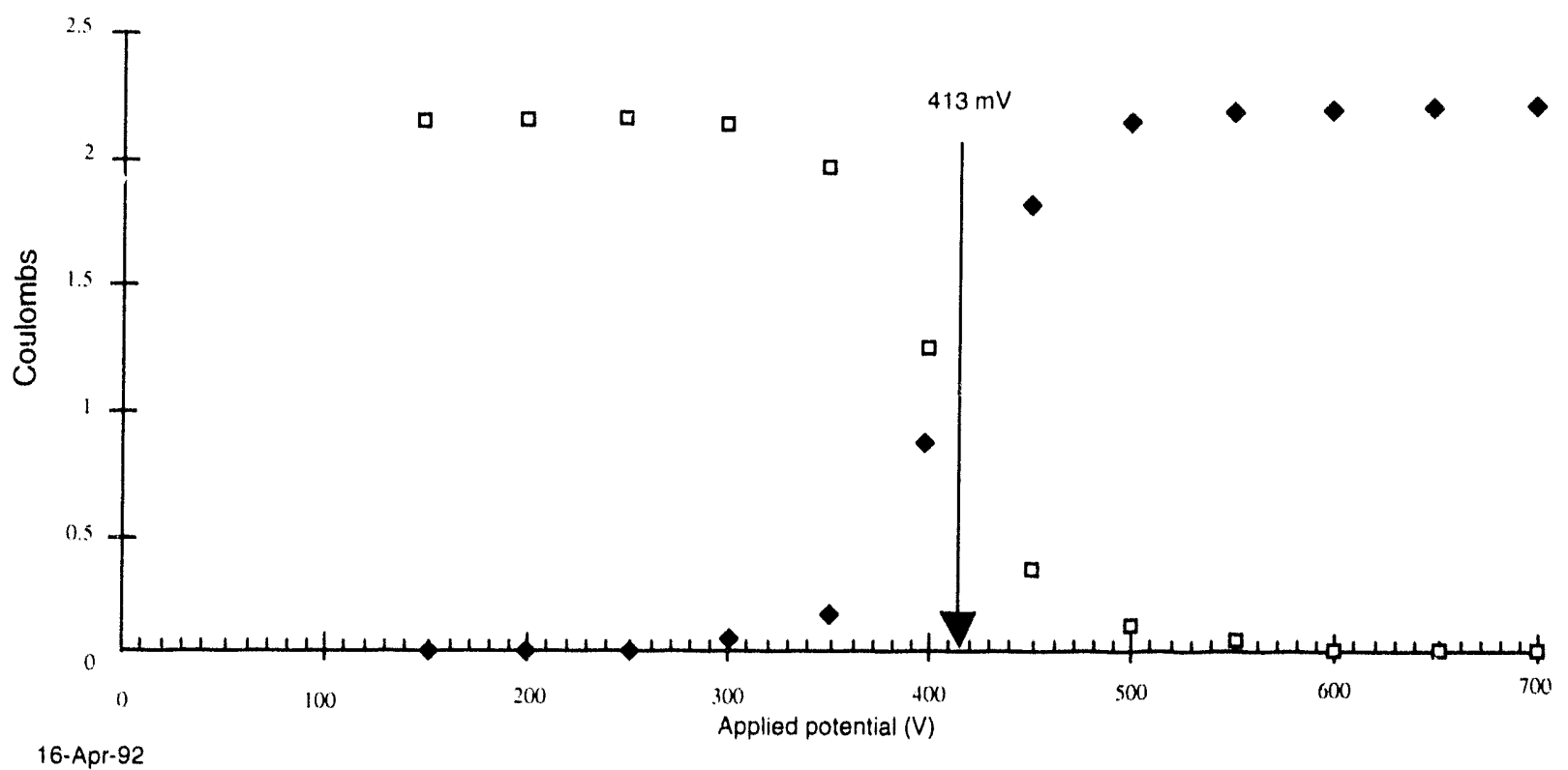

Fig. 1. Coulogram of $22.18 \mathrm{mM}$ of iron in $0.5 \mathrm{M} \mathrm{H}_{2} \mathrm{SO}_{4}$.

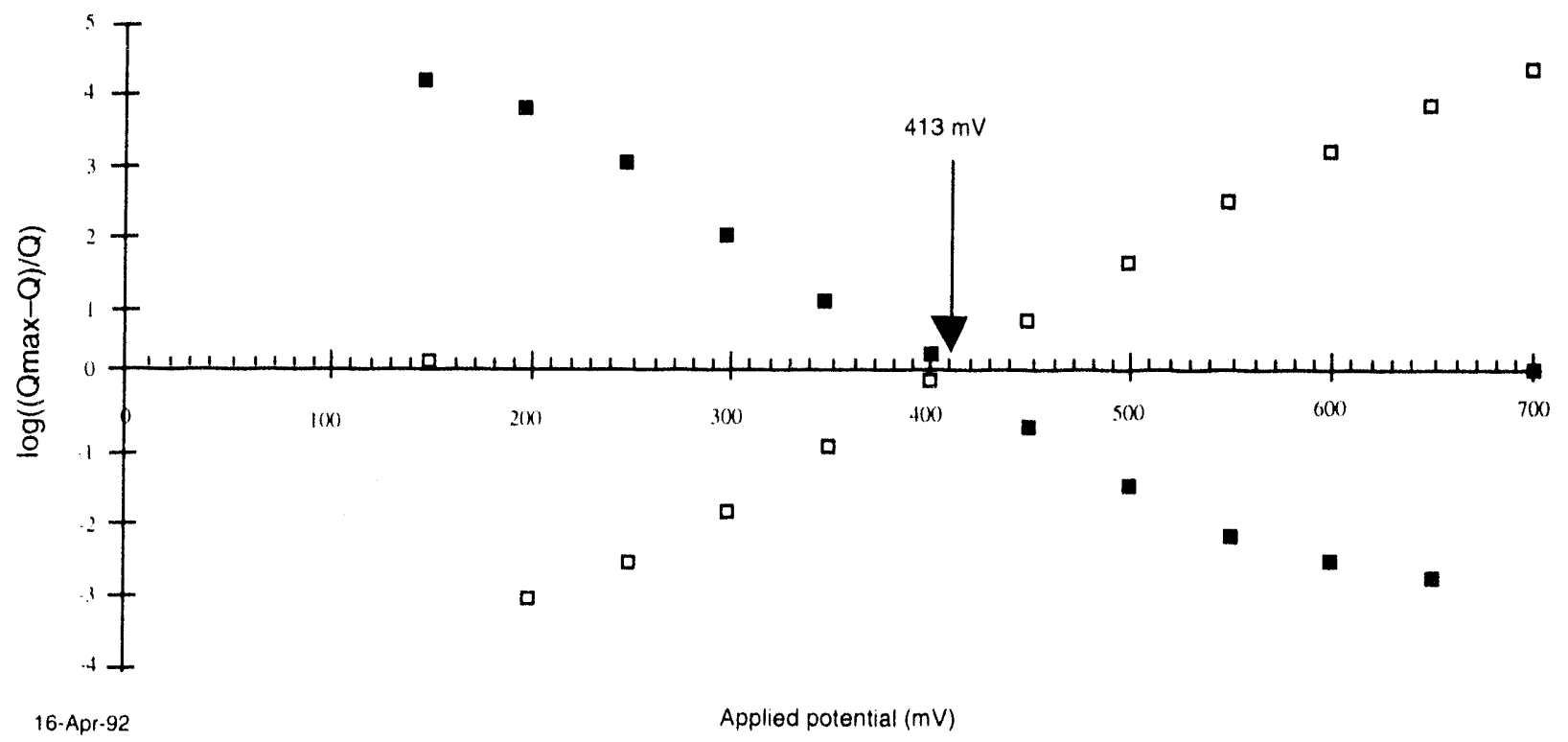

Fig. 2. Linearlized coulogram for iron.

Precision and accuracy can be examined by looking at the electrical check data and at the blank and iron solution data. Figure 3 is a plot of the measured coulombs during electrical checks. As can be seen, the data fall into three distinct regions, which are demarcated by the average line. Had the results arisen from one population, then we would expect the data to be randomly distributed around the mean. Here we see the first 24 point below the line, the next 13 above it, and the last 2 are again below it. The probability is 


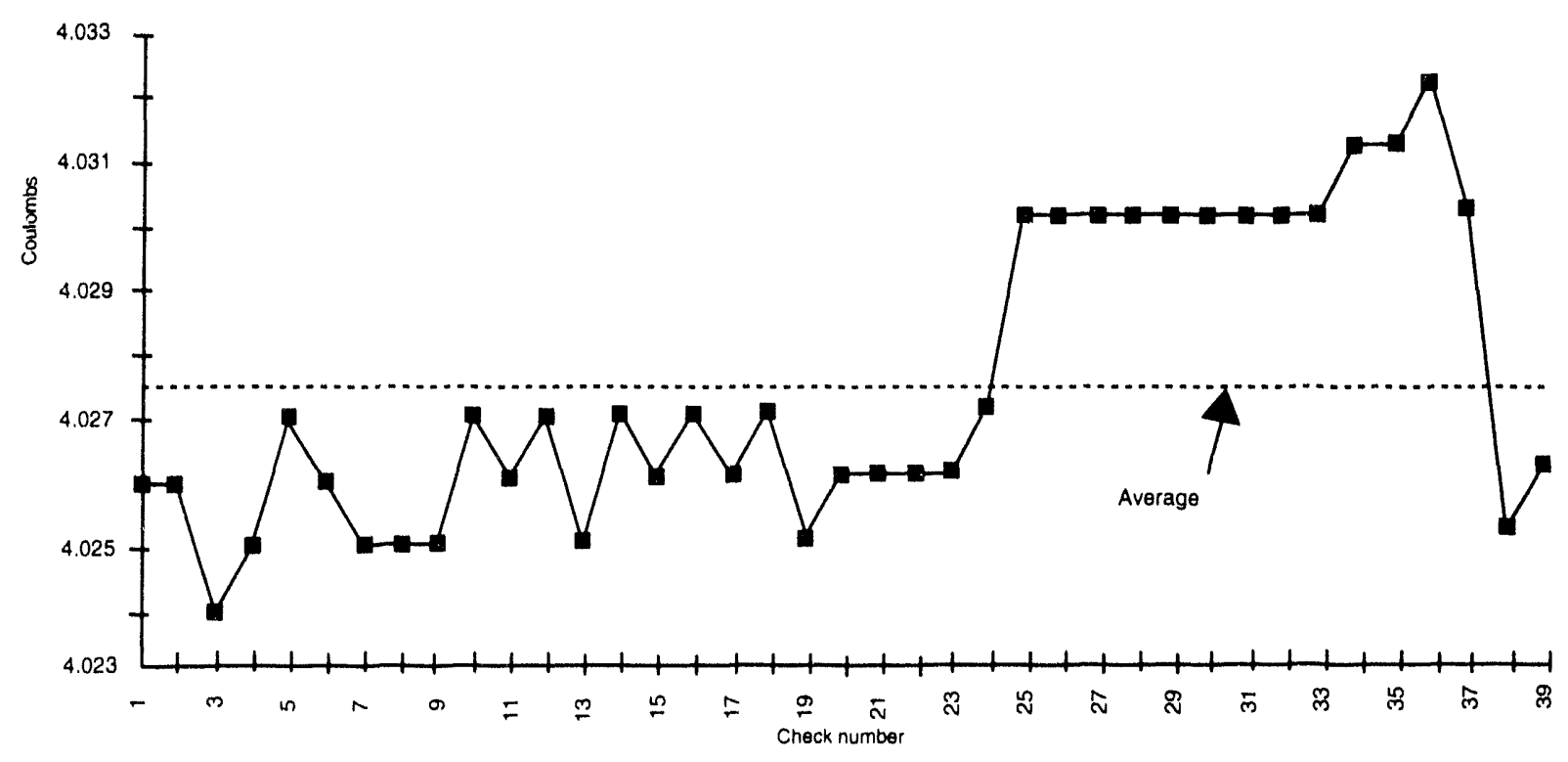

Fig. 3. Electrical checks.

very small that such a distribution of observations could occur from one population. In addition, constructing a control chart starting with the first six points and then adding to it subsequent points that fall within the 3-sigma limits reveals that after the 24th point the results are out of control. This data behavior can be attributed to various factors. The first 24 points represent the electrical checks done over a 3-week period of Part 1, the manual runs using a PAR 273. The next 13 points are the measurements made during a 2-week period 7 months later, that is, Part 2, the computer-controlled runs using a PAR 273A. The last two points were obtained also during the same time period under PARPU control but with a different PAR 279 coulometer. Table I shows the statistics for these results but omits a separate listing of the last two points because two points are not enough for significant and useful statistics.

\begin{tabular}{|c|c|c|c|}
\hline$n$ & Average & Std. Dev. & $\%$ RSD \\
\hline$\overline{39}$ & 4.02739 & 0.002232 & 0.055 \\
\hline 24 & 4.02596 & 0.000856 & 0.021 \\
\hline 13 & 4.03031 & 0.000630 & 0.016 \\
\hline
\end{tabular}

We cannot offer a definite reason for the small variation observed. We do know that the regions were generated differently, so we should not expect them to be identical. The variation may be caused by either the time difference between the two different sets of results, the difference between the two potentiostats, the presence of PARPU, a combination of all these, or some unknown factor. We cannot see how the variation could be caused by PARPU because we were careful to design the program to duplicate the manual procedure. Regardless of the cause, the overall change of $0.06 \%$ is small, and the individual variations of $0.02 \%$ over 2 - or 3 -week periods is even smaller. The fact that the results cover a range of $0.004 \mathrm{C}$ is not very significant when we recall that the analysis method is calibrated daily with chemical standards. 
Electrolysis results are shown in Table II for blanks and in Tables III and IV for iron solutions. The tabulated blank data are an extraction at $4 \mathrm{~min}$ from the $8 \mathrm{~min}$ of coulomb data collected for each blank. The results under "Average Factor" in the iron solution tabulations are obtained as the ratio of milligrams of iron electrolyzed to net coulombs measured. Net coulombs are the difference after the average blank value is subtracted. The blank values listed in 'Table II correspond to the iron results in Tables III and IV. The multiple entries in the " $\mu \mathrm{M} \mathrm{Fe"} \mathrm{(micromoles} \mathrm{of} \mathrm{iron)} \mathrm{column} \mathrm{refer} \mathrm{to} \mathrm{a} \mathrm{day's}$ run in which the amount of iron for an electrolysis was not the same but varied as listed.

\begin{tabular}{ccccc}
\hline \hline \multicolumn{5}{c}{ Table II. Electrolysis Results for Blanks } \\
\hline Part & $\boldsymbol{n}$ & Average & Std. Dev. & \% RSD \\
\hline 1 & 5 & 0.0052 & 0.0006 & 11 \\
& 3 & 0.0050 & 0.0005 & 11 \\
& 4 & 0.0053 & 0.0008 & 16 \\
& 4 & 0.0054 & 0.0005 & 8 \\
& & & & \\
1 & 4 & 0.0054 & 0.0012 & 21 \\
& 4 & 0.0055 & 0.0005 & 8 \\
& 4 & 0.0058 & 0.0006 & 11 \\
& 4 & 0.0063 & 0.0025 & 40 \\
2 & & & & \\
& 4 & 0.0105 & 0.0015 & 14 \\
& 4 & 0.0088 & 0.0010 & 11 \\
& 4 & 0.0092 & 0.0032 & 35 \\
2 & & & & \\
& 4 & 0.0115 & 0.0010 & 9 \\
\hline \hline
\end{tabular}

\begin{tabular}{cccccc}
\hline \hline \multicolumn{5}{c}{ Table III. Factors for Electrolysis Disregarding Amount of Iron Electrolyzed } \\
\hline \multirow{2}{*}{ Part } & $\mu \mathrm{M} \mathrm{Fe}$ & $\boldsymbol{n}$ & $\begin{array}{c}\text { Average } \\
\text { Factor }\end{array}$ & Std. Dev. & \% RSD \\
\hline 1 & 22 & 6 & 0.5513 & 0.0005 & 0.09 \\
& 22 & 9 & 0.5531 & 0.0004 & 0.06 \\
& 22 & 10 & 0.5521 & 0.0004 & 0.07 \\
& 22 & 10 & 0.5520 & 0.0005 & 0.09 \\
& & & & & \\
1 & 22 & 10 & 0.5524 & 0.0002 & 0.04 \\
& 22 & 10 & 0.5523 & 0.0002 & 0.04 \\
& $11,22,44$ & 15 & 0.5521 & 0.0003 & 0.06 \\
& $11,22,44$ & 15 & 0.5523 & 0.0005 & 0.09 \\
2 & 17,22 & 10 & 0.5910 & 0.0007 & 0.12 \\
& 17,22 & 10 & 0.5915 & 0.0008 & 0.14 \\
& 17,22 & 10 & 0.5916 & 0.0006 & 0.10 \\
& & & & & \\
2 & 17,22 & 10 & 0.5928 & 0.0009 & 0.15 \\
& 17,22 & 10 & 0.5926 & 0.0005 & 0.09 \\
\hline \hline
\end{tabular}




\begin{tabular}{|c|c|c|c|c|c|}
\hline \multicolumn{6}{|c|}{$\begin{array}{l}\text { Table IV. Factors for Electrolysis Considering Amount of Iron } \\
\text { Electrolyzed }\end{array}$} \\
\hline Part & $\mu \mathrm{M} \mathrm{Fe}$ & $n$ & \multicolumn{2}{|c|}{ Average } & $\%$ RSD \\
\hline \multirow[t]{3}{*}{1} & 11 & 5 & 0.5522 & 0.0004 & 0.08 \\
\hline & 22 & 5 & 0.5519 & 0.0003 & 0.05 \\
\hline & 44 & 5 & 0.5522 & 0.0002 & 0.02 \\
\hline \multirow[t]{3}{*}{1} & 11 & 5 & 0.5525 & 0.0007 & 0.12 \\
\hline & 22 & 5 & 0.5523 & 0.0005 & 0.09 \\
\hline & 44 & 5 & 0.5522 & 0.0003 & 0.05 \\
\hline \multirow[t]{2}{*}{2} & 17 & 5 & 0.5910 & 0.0008 & 0.14 \\
\hline & 22 & 5 & 0.5909 & 0.0006 & 0.11 \\
\hline \multirow[t]{2}{*}{2} & 17 & 5 & 0.5917 & 0.0011 & 0.19 \\
\hline & 22 & 5 & 0.5914 & 0.0004 & 0.07 \\
\hline \multirow[t]{2}{*}{2} & 17 & 5 & 0.5916 & 0.0009 & 0.14 \\
\hline & 22 & 5 & 0.5915 & 0.0002 & 0.04 \\
\hline \multirow[t]{2}{*}{2} & 17 & 5 & 0.5927 & 0.0013 & 0.21 \\
\hline & 22 & 5 & 0.5929 & 0.0004 & 0.07 \\
\hline \multirow[t]{2}{*}{2} & 17 & 5 & 0.5926 & 0.0004 & 0.07 \\
\hline & 22 & 5 & 0.5925 & 0.0007 & 012 \\
\hline
\end{tabular}

The factor results in Table III make no distinction between the amount of iron electrolyzed, whereas in Table IV they do.

The result of a blank or iron solution measurement incorporates not only the response of the PAR 273/279 but also of the cell to an electrolysis. The main two points we should discuss after examining the above blank and iron solution results are the differences in outcome between Parts 1 and 2. For the blanks the Part 2 results are higher, but for the iron solutions the highur factors of Part 2 represent lower amounts of anodic current. In addition, Table III shows that the precision looks better for the first part, $<0.1 \%$ compared with $0.12 \%$ for the second. The above discussion of the electrical check results can also be applied here with two differences. The different coulometer was used only for the last two electrical checks, not for any of the electrolysis runs, and the current range was not changed during electrical checks. However, we do not think the explanation for the observed differences to the blanks and factors is related to changes in the instrumentation and the control procedures.

The higher results for the blanks are probably caused by the glass-distilled water used in making up a new 8-L batch of electrolyte. We tried using electrolyte made up with ultrapure sulfuric acid, but this change did not lower the blank values. The higher factors are attributed to using a new iron stock solution after the first batch was exhausted because the factor changed for the new stock solution even though we had not changed anything else (such as potentiostats or supporting electrolyte). We are at a loss to explain 
why the iron stock solution is different. We do not have a good explanation for the lower precision of the Part 2 results, although we can saly that under such conditions precisions of $0.1 \%$ or better are attainable with at least $22 \mu \mathrm{m}$ of analyte.

During Part l, we noticed that we were getting different results from the two cells we were using. Our daily factor results had residuals that showed a consistent alternating pattern around the average. For example, the individual factors and their residuals for the item that appears on the fourth row of Table III are shown in Table V. We noticed that the sign of the pattern was related to which of the two cell vessels we used. When we removed the central contact from the vessels, we found that electrolyte had leaked into the metal-glass seal region. This behavior was not apparent affer ienoval of the central contact and sealing of the remaining hole.

\begin{tabular}{|c|c|}
\hline \multicolumn{2}{|c|}{ Table V. Residual Patter } \\
\hline Factor & Residual \\
\hline 0.5517 & $(0.0)(0) 3$ \\
\hline 0.5525 & -0.000 .5 \\
\hline 0.551 .3 & $0.00(0) 7$ \\
\hline 0.5525 & -0.0005 \\
\hline 0.551 .3 & 0.0007 \\
\hline 0.5524 & -0.0004 \\
\hline 0.5517 & 0.000 .3 \\
\hline 0.5522 & -0.0002 \\
\hline 0.5519 & 0.0001 \\
\hline 0.5525 & .0 .0005 \\
\hline
\end{tabular}

Very pertinent to this discussion of precision and accuracy is the changing of the current range that we did in Part 2. We have already mentioned that for Part 1 we left the current range at the $100-\mu \mathrm{A}$ level during all of the electrolysis. Doing this caused the $I / E$ converter to saturate, resulting in a current overload indication on the front panel of the potentiostat during most of the electrolysis. With a higher current range, such as $100 \mathrm{~mA}$ for iron solutions, no current overload occurs. However, the higher setting makes for an extremely noisy current display when the current approaches the 30- $\mu \mathrm{A}$ end-point, making the end-point undetectable. When we tried to use the automatic current-ranging option of the PAR 273, the PAR 279 results were erratic. We thus had to run the potentiostat at the 100- $\mu$ A current range. Upon further thought we decided we did not like the idea of running PAR 273's current-measuring circuit on overload during most of the electrolysis. In addition we ran an experiment, under computer control, in which we clectrolyzed 10 iron solutions, half of them at 100- $\mu \mathrm{A}$ and the other half at 10-mA current ranges. The results are shown in Table VI. A comparison of means statistical test gave a " $t$ " of 2.24, whereas the critical $t$ is 2.31 , indicating that the two sets of results are not different. Nevertheless, we still think that the instrument should not be run in an overload condition.

\begin{tabular}{ccccc}
\hline \hline \multicolumn{7}{l}{ Table VI. Varying Current Ranges } \\
\hline $\begin{array}{c}\text { Current Range } \\
(\mathbf{m A})\end{array}$ & $\boldsymbol{n}$ & $\begin{array}{c}\text { Average } \\
\text { Factor }\end{array}$ & Std. Dev. & \% RSD \\
\hline 0.1 & 5 & 0.54696 & 0.00068 & 0.12 \\
10 & 5 & 0.54808 & 0.00040 & 0.07 \\
\hline \hline
\end{tabular}


Faced with a noisy end-point current, we first tried to low-pass filter it using the PAR 273 hardware LPF or by signal averaging in PARPU. The hardware LPF was not effective because it introduced a bias into the current signal, thus making the cathodic end-point unreachable and the anodic end-point premature. Software LPF was too slow to be useful. Our other potentiostat, a PAR 273A, did not introduce a bias when the hardware LPF was used; therefore, we used it for the Part 2 work.

For even better end-point current stability, we adjusted the current range only once during the electrolysis, as we have already mentioned. Because of the erratic behavior of the PAR 279 during automatic current ranging, we carefully observed the PAR 279 during the single current range adjustment but found no apparent effect on the PAR 279.

For one set of runs we compared the results from the PAR 279 coulometer and the coulometer included in the PAR 273 potentiostat. The PAR 279 produced a factor of 0.5900 with a standard deviation of 0.00043 and an RSD of $0.073 \%$, whereas the PAR 273 's coulometer produced a factor of 0.5856 with a standard deviation of 0.00053 and an RSD of $0.091 \%$. These results increase our confidence in the usefulness of the PAR 273 's coulometer and suggest that it should be evaluated further. Perhaps it could be used instead of the PAR 279 or as a back-up or as a check of the PAR 279.

The Table IV results suggest some calibration curve properties. Over the range studied, the calibration appears to be a straight line with nonconstant variance. However, this investigation was not designed to include a rigorous calibration study, so we do not weigh our observations heavily. A calibration study should contain not only the usual measurements of curvature and homoscedastity, but because of the high precision of this instrumentation, the added complexity of variance in the independent variable, has to be included. Such considerations could be taken up in another study.

\section{B. Robustness}

Robustness is the hardiness, sturdiness, soundness, usability, or durability of the instrumentation. It refers to the practical application and use of the instrumentation, and it is just as important a requirement for our controlled-potential coulometric plutonium analysis as are precision and accuracy. Evaluation of robustness takes a pragmatic look at the newer instrumentation and therefore is concerned with reliability and ease of operation.

By far, the main reason for lack of reliability in this study was electrochemical and operator-related obstacles, not instrument shortcomings. Of the 10 difficulties encountered in Part 1,7 were electrochemical, such as long reduction times, and 3 were analyst related, such as failure to record a result. Of the 11 difficulties encountered in Part 2, 7 were electrochemical and 4 were instrumental. Most of the electrochemical difficulties are related to poisoning of the working electrode and could be mitigated by inclusion of a routine cleaning step in the procedure.

Of the four instrumental problems, two had to do with communications between the computer and the PAR 273A or PAR 279. We found that turning the cell stirrer motor on and off, as we had to do to rinse the cell, or having the "dumb" terminal connected to the PAR 273 occasionally caused the potentiostat's GPIB to set itself in the "listen" mode, locking up communications. We remedied this problem by moving the electrometer's ground clip from one of the vertical metal support rods for the cell to the cell's plastic cover and by disconnecting the dumb terminal.

Occasionally the PAR 279 would take itself off line so that the PC could not communicate with it, and the only way to reestablish the link was by cycling the coulometer off and on. This problem was very infrequent at the beginning, not 
hampering our work, but after 4 months the coulometer became totally inaccessible from the PC, forcing us to return the instrument to PAR for repair.

The filter bias has been discussed above.

The final instrumental complication arose from the fact that the PAR 273's power amplifier is capable of currents $>1$ A but the PAR 279's input circuitry cannot handle currents $>200 \mathrm{~mA}$ except when the 1 -A current range has been selected. On the rare occasions when the reference electrode bridge tube tip became partially dried, a bubble slowly formed above the tip when the tube was refilled. This bubble ended up breaking the electrical contact between the reference electrode and the cell, causing the potentiostat controller to dump large amounts of current into the cell, and into the coulometer, which destroyed the coulometer input circuitry. This problem caused the return of this coulometer for repair as well. Several software checks have been incorporated into PARPU to guard against this occurrence. One checks the stability of the reference electrode potential before starting the electrolysis while the other sets up the PAR 273 to generate an interrupt to PARPU, which is handled by turning off the cell. A third, more sure guard would be to install a $200-\mathrm{mA}$ fuse in the coulometer's input circuitry, but this defense would have to be evaluated to assure that it does not affect the instrument's response.

PARPU provides relief to the analyst in that it commits no ornissions in instrumentation setup, data recording and manipulation, or end-point current monitoring, thus improving the reliability.

Under computer control, this newer instrumentation relieved the analyst of instrumentation setup and control and of ensuring accurate data recording. To evaluate the user friendliness of PARPU, we asked two analysts not familiar with the software, but who knew the analysis, to try it. This alpha testing led to enhanced results calculations and the conclusion that PARPU was satisfactory. One difference between the old 100 series and this newer series is the larger size of the 200 series. The combination of the PAR 273 and PAR 279 doubles the volume of the potentiostat/coulometry system. When a computer - with its CPU, monitor, keyboard, and printer-are included, the volume increases by a factor of about 5 . Thus a laboratory instrumentation rack would be in order to hold all the equipment. The computer size could be decreased appreciably if a laptop and one of the small printers were used.

\section{Conclusion}

We found that the newer instrumentation satisfactorily meets the criteria we established, and thus its performance compares well with the 100 series. 


\section{APPENDIX A}

Potentiostat GPIB: 01001110 (address 14, echo on, CR terminator)

Serial: 11111001 (1 stop bit, no echo, no parity, $19.2 \mathrm{~K}$ baud, 8 bit character.)

PAR 279: 0000001 (9.6K baud)

GPIB PCIIA: 010000000 (PCIIA, 7210 mode, address 02E1, no shared interrupts)

IRQ7 (interrupt line 7)

DRQ1, DACK1 (DMA channel 1)

Shield ground selected.

GPIB.COM: Primary GPIB address: 0

Secondary GPIB address: none

Time-out setting: $\mathrm{T} 10 \mathrm{~s}$

EOS byte: $0 \mathrm{DH}$

Terminate string on EOS: no

Set EOI with EOS on write: no

Type of compare on EOS: 7 bit

Set EOI w/last byte of Write: yes

System controller: yes

Assert REN when SC: no

Enable auto serial polling: no

Timing: $500 \mathrm{~ns}$

Enable 488.2 protocols: yes

CIC protocol: no

Handler type: $\mathrm{pc} 2 \mathrm{a}$

Interrupt setting: 7

Base I/O address: 02E $1 \mathrm{H}$

DMA channel (arbitration): NONE

The device characteristics are

Primary GPIB Address: 14

Secondary GPIB address: none

Time-out setting: T10s

EOS byte: $00 \mathrm{H}$

Terminate read on EOS: no

Set EOI w/last byte on Write: yes

Repeat addressing: no

CONFIG.SYS: Has to have a DEVICE=GPIB.COM statement.

The GPIB.COM was modified using the National Instruments (NI) IBCONF

program. The NI QBDECL.BAS file had to be accessible to PARPU. A library file was made by linking the NI QBIB.OBJ with BQLB45.LIB. To run PARPU from the QuickBASIC 4.5 environment, the DOS command line included QB PARPU $/ \mathrm{L}$ 
QBIB.QLB. An executable file was made by linking the compiled PARPU with QBIB.OBJ and supplying the BCOM45.LIB + QBIB.LIB library names.

The QuickBASIC program, called "PARPU", connects to the PAR 273 through a GPIB port and to the PAR 279 through an RS232 port. Data are reported to the screen, to a printer, and to disk.

The user-PARPU interaction occurs by means of the monitor screen, keyboard, and audible beeps or alarms. Four screens display an introductory logo, a main menu, an electrolysis graphical output, and a results calculation option. The main menu screen displays function-selectable options, parameter settings of the PAR 273, PAR 279 and the program, and status lines. Currently 8 options are available to the analyst:

\author{
1-3. Configure system for blank, sample, or \\ electrical check. \\ 4. Modify default parameters of the three \\ configurations. \\ 5. Start electrolysis. \\ 6. Individual command control of the 273. \\ 7. Abort or quit program. \\ 8. DOS shell.
}

The parameters displayed are the reduction and oxidation potentials, the delays at each of these potentials, the scan rate (a potential step), and PAR 273 current range. The PAR 279 parameters presented are current range, anodic or cathodic function, and whether to place the PAR 279 on or off line. The PARPU parameters are amperometric end-point current level, whether the electrolysis is terminated by an end-point or time limit, the option to print the results, and end-point current signal averaging index.

The status lines display configuration selected, error messages, and whether the $\mathrm{PC}$ is configuring the PAR instruments.

The electrolysis screen graphs the progress of the electrolysis as current vs time, displays the current and time read-out, and the coulombs read from the PAR 279 after electrolysis is completed. From this screen the program or run can be terminated, data can be saved to disk, data can be printed, and sample variables, such as weight, can be entered. The data that are saved and printed consist of the date and time, run type (blank or sample identification), and reduction and oxidation times and coulombs measured by the PAR 273 and PAR 279. Saved data are in a spreadsheet-readable form.

Each run type is handled slightly differently. For a blank, reduction occurs to an amperometric end-point. Oxidation, on the other hand, occurs for a preselected time, regardless of the current, and the coulombs are read from the PAR 279 and PAR 273 every minute. These incremental oxidation data are also printed and saved. For a sample, both reduction and oxidation are run to an amperometric end-point. For a standard sample a factor is calculated, whereas for an unknown sample the percentage of plutonium is calculated using an analyst-selectable factor. For an electrical check, only the oxidation is run for a preselected time.

Error prevention/recovery is by no means universal, although a few contingencies have been addressed. An eager operator pushing the function keys cannot cause nested SUBing. Verification of the PAR 273 parameter settings is performed, although as of now there is no recovery from an incorrect parameter. Printer and disk drive oversights, such as the printer off-line or no floppy in the drive are handled. The reference electrode 
is monitored, and if it is found unstable the electrolysis cannot commence. The PAR 273 overpotential state is monitored as well as the position of the user-activated manual cellenable switch and the electrometer's dummy cell switch. The overpotential condition is handled by immediately turning of ${ }^{c}$ the cell. The PAR 279 current range setting is inaccessible to computer control, so an annoying, blinking reminder is shown on the screen to prompt the analyst.

\section{REFERENCES}

1. Ryzhinskii, M.V., Bulyanitsa, L.S., Nikitina, S.A. Radiokhimiya 22(6), 785-811, 1980.

2. Lebedev, I.A., Myasoedov, B.F. Radiokhimiya 24 (6), 700-728, 1982.

3. Myasoedov, B.F., Markov, V.K. Radiokhimiya 26 (4), 505-522, 1984.

4. ASTM method C1165-90.

5. Harrar, Jackson E., Electroanalytical Chemistry A Series of Advances, Allen J. Bard, Ed., Vol. 8 (Marcel Dekker, Inc., New York, 1975). 

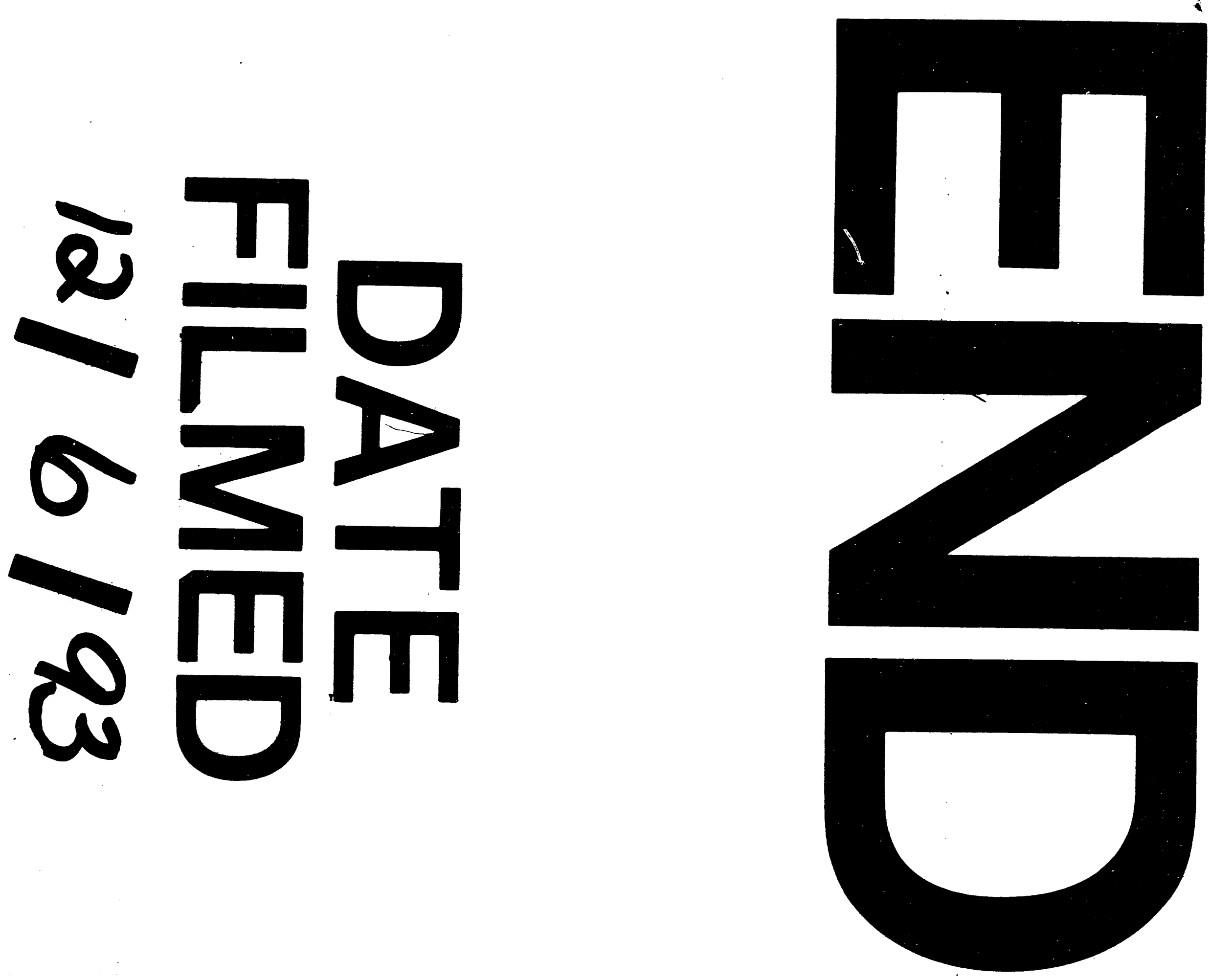
$\ldots$ 